University of Wollongong

Research Online

Faculty of Engineering - Papers (Archive)

Faculty of Engineering and Information

Sciences

2009

\title{
Fast and accurate proton computed tomography image reconstruction for applications in proton therapy
}

\author{
Scott Penfold \\ University of Wollongong, snp75@uow.edu.au
}

R. W. Schulte

Loma Linda University, US

V. Bashkirov

Loma Linda University, US

Anatoly B. Rosenfeld

University of Wollongong, anatoly@uow.edu.au

Follow this and additional works at: https://ro.uow.edu.au/engpapers

Part of the Engineering Commons

https://ro.uow.edu.au/engpapers/510

\section{Recommended Citation}

Penfold, Scott; Schulte, R. W.; Bashkirov, V.; and Rosenfeld, Anatoly B.: Fast and accurate proton computed tomography image reconstruction for applications in proton therapy 2009, 213-216.

https://ro.uow.edu.au/engpapers/510 


\title{
Fast and Accurate Proton Computed Tomography Image Reconstruction for Applications in Proton Therapy
}

\author{
S.N. Penfold ${ }^{1}$, R.W. Schulte ${ }^{2}$, V. Bashkirov ${ }^{2}$, and A.B. Rosenfeld ${ }^{1}$ \\ ${ }^{1}$ Centre for Medical Radiation Physics, University of Wollongong, Wollongong, Australia \\ ${ }^{2}$ Department of Radiation Medicine, Loma Linda University Medical Center, Loma Linda, USA
}

\begin{abstract}
Proton computed tomography has been suggested as a means for maximizing the potential benefits of proton radiation therapy. By measuring individual proton energy losses after traversing an object and predicting paths of maximum likelihood through the image space, relative stopping power maps can be generated for treatment planning and image guidance. However, the processes of proton interaction with the imaged object lead to a number of challenges in the image reconstruction procedure. In this work we describe our approach to obtaining accurate relative stopping power maps in the shortest amount of time.
\end{abstract}

Keywords-proton computed tomography, relative stopping power, block-iterative projection.

\section{INTRODUCTION}

The majority of current proton treatment centers use pencil beam algorithms for treatment planning, due to their computational efficiency. In this approach, a dose deposition model based on experimentally measured depth dose curves in water is convolved with a $3 \mathrm{D}$ map of relative stopping powers [1]. Currently, the relative stopping powers are obtained by converting X-ray CT Hounsfield units via an empirically derived calibration curve [2],[3]. This conversion can lead to errors in the estimated proton range during treatment, however [4].

Proton CT (pCT) offers the possibility of directly obtaining relative stopping powers from proton energy loss measurements, removing the errors associated with the Hounsfield conversion methods. In one of the current generation pCT designs [5], individual protons are tracked pre- and post-patient with 2D sensitive silicon strip detectors (SSDs), providing information about proton position and direction at the boundaries of the image space. This allows the effects of multiple Coulomb scattering within the object to be accounted for in a most likely path (MLP) estimation [6],[7].

In addition to tracking the position of individual protons, the energy lost by each proton after traversal of the image space is recorded. Using these measurements, one can calculate either the path integral of relative electron density of a water equivalent object [5], i.e., an object of water composition but varying electron density that produces the same energy loss as the real object, or the integral of relative stopping power along each proton path. In this study we calculated the latter with Eq. 1.

$$
\int_{L} \rho_{s}(\mathbf{r}) d l=\int_{E_{\text {out }}}^{E_{\text {in }}} \frac{d E}{S\left(I_{\text {water }}, E\right)}
$$

In Eq. 1, $E_{\text {in }}$ and $E_{\text {out }}$ are the measured entry and exit proton energies at the image space boundaries respectively, $\rho_{s}$ is the stopping power relative to water at spatial location $\mathbf{r}$, and $L$ is the estimated proton path through the image space. The stopping power in water $S\left(I_{\text {water }}, E\right)$ is given by the Bethe-Bloch equation (Eq. 2).

$$
S\left(I_{\text {water }}, E\right)=\frac{4 \pi r_{e} m_{e} c^{2} N_{e, \text { water }}}{\beta^{2}(E)}\left[\ln \left(\frac{2 m_{e} c^{2}}{I_{\text {water }}} \frac{\beta^{2}(E)}{1-\beta^{2}(E)}\right)-\beta^{2}(E)\right]
$$

Here, $r_{e}$ is the classical electron radius, $m_{\mathrm{e}}$ is the mass of the electron, $N_{e, w a t e r}$ is the electron density of water, $\beta$ is the velocity of the proton relative to the speed of light $c$, and $I_{\text {water }}$ is the mean excitation energy of water. By recording many line integrals from different projection angles, threedimensional relative stopping power maps of the patient can be generated.

Iterative projection methods, e.g., the algebraic reconstruction technique (ART) [8], have been demonstrated as the preferred way of accommodating the nonlinear proton paths [7]. Such methods are based on solving a system of equations of the form

$$
\mathbf{A x}=\mathbf{b},
$$

where $\mathbf{A}$ is an $n \times m$ matrix whose elements $a_{j}^{i}$ correspond to the length of intersection (chord length) of the $i$-th proton history's path with the $j$-th voxel, $\mathbf{x}$ is the unknown $m$ dimensional relative stopping power image vector, and $\mathbf{b}$ is the $n$-dimensional vector, whose elements $b_{i}$ correspond to the integral relative stopping power along the $i$-th proton path derived from the energy loss measurements (Eq. 1).

In GEANT4 simulations of a head phantom, we have observed that when the chord lengths are set at a constant value, equal to the pixel size, the reconstructed images systematically underestimated the actual relative stopping 
power of the imaged object by up to $13 \%$. This would limit the usefulness of pCT-generated quantitative stopping power maps in treatment planning calculations.

Another challenge of clinical pCT is that very fast image reconstruction is required. ART carries out image updates sequentially after each proton history and is therefore inherently serial, meaning that the speed of the reconstruction is dependent on the speed of the computer processing unit. Considering the huge number of collected proton histories, which is of the order of $10^{8}$ for a head-size object, reconstructions with ART are on the order of hours, even with the fastest processing units. Such long reconstruction times would exclude the usefulness of pCT for image guidance in the treatment room.

With the development of parallel computing, work has been dedicated to developing iterative projection algorithms that can be executed in parallel over multiple processors to enable fast algebraic reconstructions. We have previously shown [10] that the method of diagonally relaxed orthogonal projections (DROP) [9], which is parallelizable, required less iteration than ART to produce optimal images. However, these results were produced under the assumption of constant chord lengths as described above.

In this work we describe a method for calculating a variable mean chord length factor for Eq. 3 that depends on the angle of the proton beam relative to the reconstruction grid, and demonstrate how this improves the accuracy of quantitative pCT. Also, the performance of the DROP method in comparison to ART, with the variable mean chord length factor, is investigated.

\section{Methods And MATERIALs}

\section{A. Effective Mean Chord Length}

Our previous approach to $\mathrm{pCT}$ reconstruction has been to calculate the MLP of each proton based on entry and exit information and to determine which pixels are intersected. The elements of $\mathbf{A}$ in Eq. 3 belonging to these pixels were then assigned a constant chord length, while the elements corresponding to non-intersected pixels were assigned a value of zero.

To obtain a more accurate mean chord length that varies with beam rotation angle, a computer program generating a large number of random straight-line chords was created. A conceptual illustration of this process is shown in Fig. 1, where two extreme cases of proton path orientation relative to the pixel are considered ( 0 and 45 degrees).

Mean chord lengths were determined for pixel rotation angles from 0-89 degrees in 1 degree increments, corresponding to the orientation of the multiple projection angles with respect to the fixed image reconstruction grid. From a

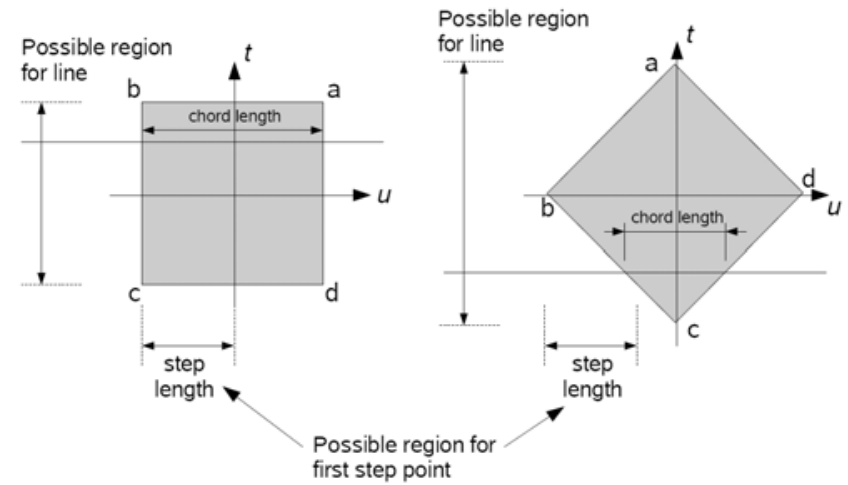

Fig. 1 Schematic of the program created to calculate the effective mean chord length of a line parallel to the $\mathrm{u}$-axis and a rotated pixel

randomly selected initial step-point (see Fig. 1), half pixel step lengths were used to determine if step-points on a chord line parallel to the $u$-axis, with random $t$ intersection would fall within the pixel boundaries. Note that each line intersected the pixel to some degree. If a step-point was within the pixel boundaries, the chord length was stored in a "detected" variable and if it was outside the pixel boundaries, the chord length was stored in a "undetected" variable.

For each pixel rotation angle, a total of 100,000 chord lines were generated and a mean chord length was calculated for both the detected and undetected fractions of chords. An effective mean chord length was then defined as follows (Eq. 4):

$$
l_{\text {eff }}=l_{\text {det }}+p_{\text {undet }} l_{\text {undet }}
$$

Here $l_{\text {det }}$ and $l_{\text {undet }}$ are the mean chord lengths of detected and undetected lines, respectively, and $p_{\text {undet }}$ is the fraction of lines that went undetected. The function of effective mean chord length versus pixel rotation angle was approximated by fitting a $4^{\text {th }}$ degree polynomial to the data.

\section{B. GEANT4 Simulation and Reconstruction with ART}

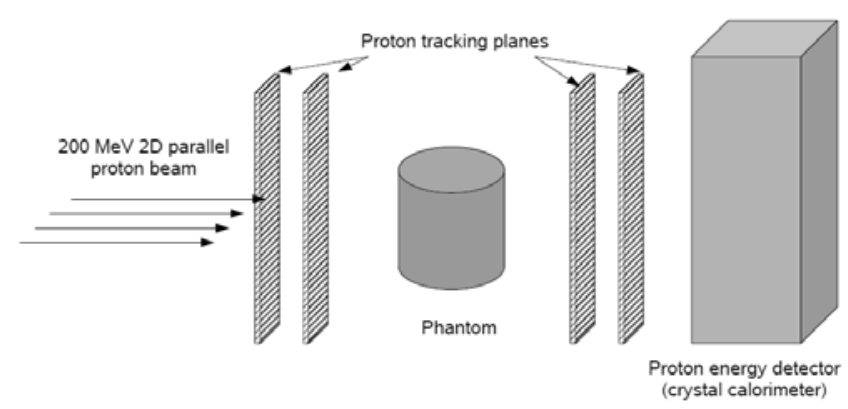

Fig. 2 Geometry of the GEANT4 proton CT simulation 
To test the application of this effective mean chord length concept to quantitative pCT image reconstruction, a Monte Carlo radiation transport simulation of a pCT system was created (Fig. 2) using the GEANT4 toolkit [11]. Four $30 \times 30 \mathrm{~cm}^{2} 2 \mathrm{D}$ sensitive silicon tracking planes were assigned a spatial resolution of $100 \mu \mathrm{m}$. The energy detector was simulated as a cesium iodide rectangular prism with perfect energy resolution.

A cylindrical head phantom with an elliptic cross-section (see Fig. 3a), based on the phantom of Herman [12], was positioned at the center of the imaging system. The bone and brain regions were assigned the chemical composition given by ICRP Report 23 [13]. The ventricular regions, corresponding to cerebro-spinal fluid, were assigned the chemical composition of water. The true relative stopping power of the different regions was calculated with Eq. 5 .

$$
\rho_{s}=\frac{1}{\Delta E} \int_{10}^{200} \eta_{e, m} \frac{\left[\ln \left(\frac{2 m_{e} c^{2}}{I_{m}} \frac{\beta^{2}(E)}{1-\beta^{2}(E)}\right)-\beta^{2}(E)\right]}{\left[\ln \left(\frac{2 m_{e} c^{2}}{I_{\text {water }}} \frac{\beta^{2}(E)}{1-\beta^{2}(E)}\right)-\beta^{2}(E)\right]} d E
$$

Here, $\eta_{e, m}$ and $I_{m}$ are the electron density relative to water and mean excitation energy of the region material, respectively. The fraction in the integral varies only slowly with energy above $10 \mathrm{MeV}$, so an average over the energy range $\Delta E=200-10 \mathrm{MeV}$ was taken.

A 2D parallel beam of $200 \mathrm{MeV}$ protons was used as primary particles with the first 20,000 proton histories to traverse the system and deposit energy in the CsI scintillator in each projection angle being recorded. A total of 180 projection angles at 2 degree intervals were carried out.

The GEANT4 standard model for hadronic ionization was implemented with $d E / d x$ values being calculated in 2000 bins ranging from $1 \mathrm{keV}$ to $500 \mathrm{MeV}$. Elastic and inelastic nuclear collisions were also enabled.

Images of the Herman head phantom were reconstructed (i.e., iteratively finding a solution to Eq. 3) with ART (Eq. $6)$. Here, $k$ is the iteration index, $x^{k}$ and $x^{k+1}$ are the current and updated image estimates respectively, $a^{i}$ is the $i$-th row vector of $\mathbf{A}, b_{i}$ is as described above, and $\lambda$ is a user determined relaxation parameter.

$$
x^{k+1}=x^{k}+\lambda \frac{b_{i}-\left\langle a^{i}, x^{k}\right\rangle}{\left\|a^{i}\right\|^{2}} a^{i}
$$

For one complete cycle of reconstruction, the index $i$ runs from 1 to $m$, the number of proton histories in the data set.

\section{Reconstruction with a Block-Iterative Algorithm}

The block-iterative DROP algorithm [9] is given in Eq. 7. Here, $j$ is the pixel index, $B_{t}$ is the set of proton histories assigned to the $t$-th block, $\tau_{j}$ is the number of proton histories found to intersect the $j$-th pixel in block $t$, and all other variables are as described above. The inner summation term on the right hand side of Eq. 7 allows for parallel computations.

$$
x_{j}^{k+1}=x_{j}^{k}+\frac{\lambda}{\tau_{j}} \sum_{i \in B_{t}} \frac{b_{i}-\left\langle a^{i}, x^{k}\right\rangle}{\left\|a^{i}\right\|^{2}} a^{i}
$$

Reconstructions were done with the same projection data as in Section II $B$, with the data subdivided into 60 equal blocks. The relative error (Eq. 8) of the reconstructed image after each cycle was calculated and used as a means of image quality evaluation. Here, $x_{j}^{\prime}$ is the phantom relative stopping power in pixel $j$ and $x_{j}^{k}$ is the reconstructed relative stopping power after $k$ cycles in pixel $j$.

$$
\varepsilon_{k}=\sum_{j}\left|x_{j}^{\prime}-x_{j}^{k}\right| / \sum_{j}\left|x_{j}^{\prime}\right|
$$

\section{Results}

\section{A. Relative Stopping Power Reconstruction}

The GEANT4 pCT simulation data was reconstructed using the effective mean chord length defined in Eq (4) and also with the chord length set to a constant value equal to the pixel size. Images reconstructed up until the $10^{\text {th }}$ cycle through the data were recorded. The optimal image for each approach within 10 cycles, in terms of relative error (Eq. 8), is shown in Fig. 3.

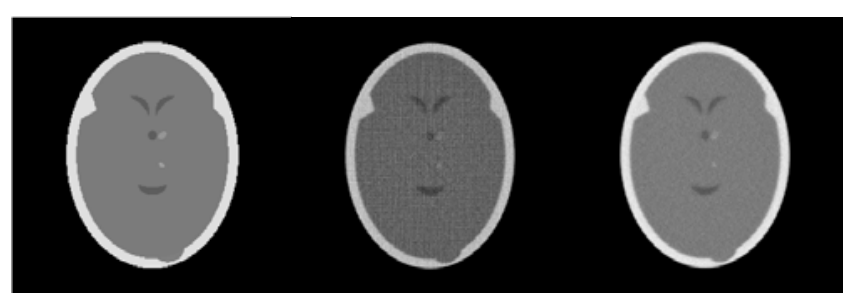

a)

b)

c)

Fig. 3 Head phantom and the images reconstructed with ART. a) Phantom, b) constant chord length and c) effective mean chord length

Histograms of the pixel values within the bone and brain regions were generated. Table 1 contains the results of Gaussian fits to the histogram plots. The mean brain reconstructed value lies within $0.15 \%$ of the phantom value and the mean bone reconstructed value within $0.7 \%$ when the effective mean chord length is used. This compares 
favorably to the corresponding values of $10 \%$ and $11 \%$ when a constant chord length is used.

Table 1 Results of Gaussian fit to bone and brain region histograms

\begin{tabular}{cccccc}
\hline \multirow{2}{*}{ Region } & Phantom & \multicolumn{2}{c}{ Constant Chord Length } & \multicolumn{2}{c}{ Mean Chord Length } \\
& $\rho_{s}$ & mean & $\sigma$ & mean & $\sigma$ \\
\hline Brain & 1.0315 & $0.928 \pm 0.001$ & 0.0524 & $1.033 \pm 0.001$ & 0.025 \\
Bone & 1.4613 & $1.307 \pm 0.002$ & 0.0527 & $1.471 \pm 0.001$ & 0.033
\end{tabular}

\section{B. Block-Iterative Performance}

Figure 4 illustrates the relative error (Eq. 8) as a function of cycle number for the ART and DROP algorithms when employing the effective mean chord length factor. DROP reached an optimal image after only 4 cycles through the data while ART required 8 cycles. Work is currently being undertaken to execute DROP on general purpose graphical processing units (GPGPU's). Preliminary results suggest that the combination of the parallelizable algorithm and the GPGPU hardware will allow for reconstruction of these pCT images in less than 10 minutes.

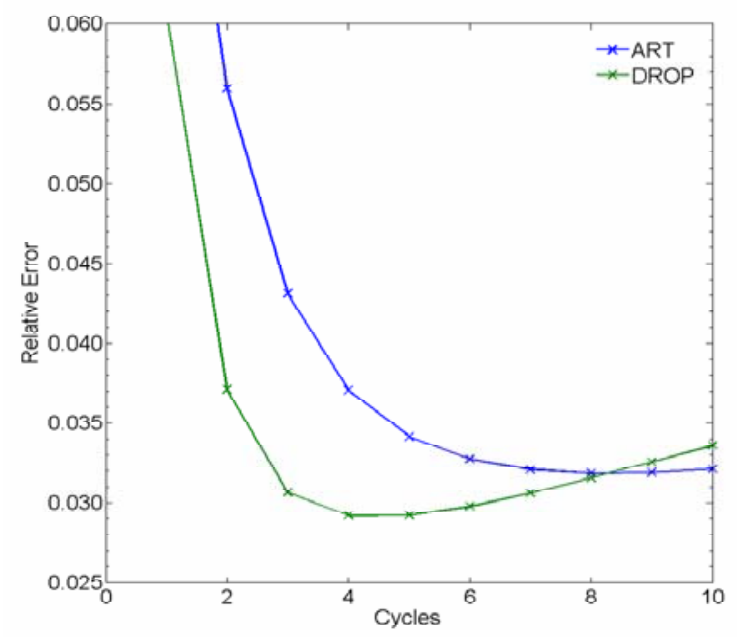

Fig. 4 Relative error of reconstructed images as a function of cycle number for ART and DROP using effective mean chord length factor

\section{Conclusions}

Proton computed tomography is an imaging modality that can directly provide the data required for proton treatment planning. We have described a method that incorporates projection angle-dependent pixel chord lengths in the iterative reconstruction process. This led to more accurate quantitative reconstructed images. Also, we have demonstrated that employing this approach in a blockiterative reconstruction algorithm (DROP) results in a smaller image error and faster convergence in comparison to the classic ART algorithm. This should lead to pCT images being reconstructed in clinically relevant time frames.

\section{AcKnowledgment}

This work was supported by Grant Number 08/RSA/1-02 from the Cancer Institute NSW.

\section{REFERENCES}

1. Hong L, Goitein M, Bucciolini M et al. (1996) A pencil beam algorithm for proton dose calculations. Phys. Med. Biol. 41:1305-1330

2. Mustafa A A and Jackson D F (1983) The relation between X-ray CT numbers and charged particle stopping powers and its significance for radiotherapy treatment planning. Phys. Med. Biol. 28:169-176

3. Schneider U, Pedroni E and Lomax A (1996) The calibration of CT Hounsfield units for radiotherapy treatment planning. Phys. Med. Biol. 41:111-124

4. Schaffner B and Pedroni E (1998) The precision of proton range calculations in proton radiotherapy treatment planning: experimental verification of the relation between CT-HU and proton stopping power. Phys. Med. Biol. 43:1579-1592

5. Schulte R W, Bashkirov V, Li T et al. (2004) Conceptual design of a proton computed tomography system for applications in proton radiation therapy. IEEE Trans. Nuc. Sci., 51:866-872

6. Schulte R W, Penfold S N, Tafas J T et al. (2008) A maximum likelihood proton path formalism for application in proton computed tomography. Med. Phys. 35:4849-4856

7. Li T, Liang Z, Singanallur J V et al. (2006) Reconstruction for proton computed tomography by tracing proton trajectories: A Monte Carlo study. Med. Phys. 33:699-706

8. Gordon R, Bender R and Herman G T (1970) Algebraic reconstruction techniques (ART) for three-dimensional electron microscopy and x-ray photography. Jour. Theor. Biol. 29:471-481

9. Censor Y, Elfving T, Herman G T et al. (2008) On diagonally-relaxed orthogonal projection methods. SIAM Jour. Sci. Comp. 30:473-504

10. Penfold S N, Schulte R W, Censor Y et al. (2009) Block-iterative and string-averaging projection algorithms in proton computed tomography image reconstruction. In "Biomedical Mathematics: Promising Directions in Imaging, Therapy Planning and Inverse Problems", Medical Physics Publishing, in press.

11. Agostinelli S, Allison J, Amako K et al. (2003) GEANT4 -A simulation toolkit. Nucl. Instrum. Methods Phys. Res. A. 506:250-303

12. Herman G T (1980) Image reconstruction from projections: the fundamentals of computerized tomography. Academic Press, NY

13. ICRP 1975 Report of the Task Group on Reference Man ICRP Publication 23

Author: Scott Penfold

Institute: Centre for Medical Radiation Physics

Street: Northfields Ave

City: Wollongong

Country: Australia

Email: snp75@uow.edu.au 